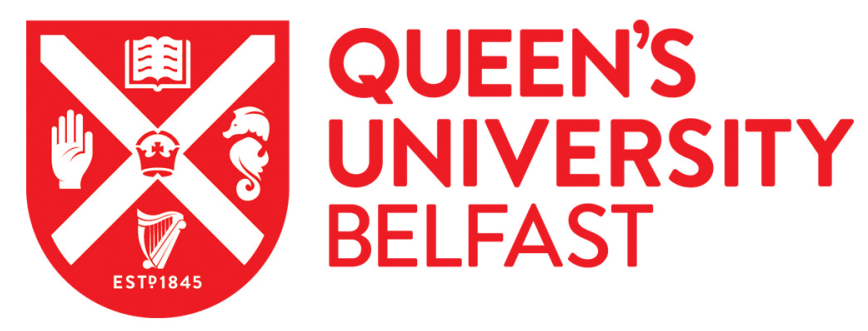

\title{
A Dual Distributed Optimal Energy Management Method for Distribution Grids With Electric Vehicles
}

Hoang, P. H., Ozkan, G., Badr, P. R., Papari, B., Edrington, C. S., Zehir, M. A., Hayes, B., Mehigan, L., Kez, D. A., \& Foley, A. M. (2021). A Dual Distributed Optimal Energy Management Method for Distribution Grids With Electric Vehicles. IEEE Transactions on Intelligent Transportation Systems. https://doi.org/10.1109/TITS.2021.3126543

Published in:

IEEE Transactions on Intelligent Transportation Systems

Document Version:

Peer reviewed version

Queen's University Belfast - Research Portal:

Link to publication record in Queen's University Belfast Research Portal

Publisher rights

Copyright 2021, IEEE.

This work is made available online in accordance with the publisher's policies. Please refer to any applicable terms of use of the publisher.

\section{General rights}

Copyright for the publications made accessible via the Queen's University Belfast Research Portal is retained by the author(s) and / or other copyright owners and it is a condition of accessing these publications that users recognise and abide by the legal requirements associated with these rights.

Take down policy

The Research Portal is Queen's institutional repository that provides access to Queen's research output. Every effort has been made to ensure that content in the Research Portal does not infringe any person's rights, or applicable UK laws. If you discover content in the Research Portal that you believe breaches copyright or violates any law, please contact openaccess@qub.ac.uk. 


\section{- IEEE $\mid \begin{aligned} & \text { Transactions on Intelligent } \\ & \text { Transportation Systems }\end{aligned}$}

\section{A Dual Distributed Optimal Energy Management Method for Distribution Grids with Electric Vehicles}

\begin{tabular}{|c|c|}
\hline Journal: & Transactions on Intelligent Transportation Systems \\
\hline Manuscript ID & T-ITS-20-09-1875.R1 \\
\hline Manuscript Type: & Regular Papers \\
\hline $\begin{array}{r}\text { Date Submitted by the } \\
\text { Author: }\end{array}$ & 26-Apr-2021 \\
\hline Complete List of Authors: & $\begin{array}{l}\text { Hoang, Phuong; Clemson University, Electrical and Computer } \\
\text { Engineering } \\
\text { Ozkan, Gokhan; Clemson University, Electrical and Computer } \\
\text { Engineering } \\
\text { Ramezani Badr, Payam; Clemson University, Electrical and Computer } \\
\text { Engineering } \\
\text { Papari, Behnaz; University of North Carolina at Charlotte, Search } \\
\text { Results Web Result with Site Links Electrical and Computer Engineering } \\
\text { Edrington, Christopher; Clemson University, Electrical and Computer } \\
\text { Engineering } \\
\text { Zehir, Aparslan; University College Cork, Energy Policy and Modelling } \\
\text { Group, MaREI Centre, Environmental Research Institute } \\
\text { Mehigan, Laura; University College Cork, Energy Policy and Modelling } \\
\text { Group, MaREI Centre, Environmental Research Institute } \\
\text { Hayes, Barry; University College Cork, School of Engineering } \\
\text { Kez, Dlzar; Queen's University Belfast, School of Electronics, Electrical } \\
\text { Engineering and Computer Science } \\
\text { Foley, Aoife; Queen's University Belfast, School of Mechanical and } \\
\text { Aerospace Engineering and School of Electronics, Electrical Engineering } \\
\text { and Computer Science }\end{array}$ \\
\hline Keywords: & $\begin{array}{l}\text { distributed energy management, electric vehicle, controller-hardware-in- } \\
\text { the-loop simulation, real-time simulation, distribution grid }\end{array}$ \\
\hline Abstract: & $\begin{array}{l}\text { Future distribution grids are expected to face an increasing penetration } \\
\text { of electric vehicles (EVs) and heterogeneous distributed energy } \\
\text { resources (DERs). This demands a distributed energy management (EM) } \\
\text { to manage power generation and delivery of energy sources to maintain } \\
\text { power quality under the impact of EV charging, to save operating costs, } \\
\text { and to enhance resiliency. However, the global optimality of the } \\
\text { distributed EM's optimization problem is still an issue in existing work } \\
\text { because of the non-convex nature of the optimization problem. In this } \\
\text { paper, a distributed EM strategy for grid-connected distribution networks } \\
\text { is proposed. In particular, the EM strategy is composed of two steps. In } \\
\text { the first step, some conditions of the EM optimization task are relaxed to } \\
\text { apply an algorithm converging to the global optimality. The results of the } \\
\text { first step are used to reconfigure constraints of the full optimization } \\
\text { problem in Step } 2 \text {. The proposed scheme is validated by implementing } \\
\text { the real-time controller-hardware-in-the-loop (CHIL) experimentation on } \\
\text { the IEEE } 33 \text { bus system. To study the impact of EV charging, EV data is }\end{array}$ \\
\hline
\end{tabular}


collected from the market and the literature to generate realistic EV load profiles to demonstrate the effectiveness of the proposed strategy on saving operating costs and maintaining power quality.

\section{SCHOLARONE ${ }^{\text {m }}$ Manuscripts}




\title{
A Dual Distributed Optimal Energy Management Method for Distribution Grids with Electric Vehicles
}

\author{
Phuong H. Hoang*, Student Member, IEEE, Gokhan Ozkan*, Member, IEEE, \\ Payam Ramezani Badr*, Student Member, IEEE, Behnaz Papari ${ }^{\dagger}$, Member, IEEE, \\ Christopher S. Edrington*, Senior Member, IEEE, Mustafa Alparslan Zehir ${ }^{\ddagger}$, Member, IEEE, \\ Barry Hayes $^{\perp}$,Senior Member, IEEE, Laura Mehigan ${ }^{\ddagger}$, Student Member, IEEE, \\ Dlzar Al Kez ${ }^{\S}$, Student Member, IEEE, Aoife M. Foley ", Member, IEEE
}

\begin{abstract}
Future distribution grids are expected to face an increasing penetration of electric vehicles (EVs) and heterogeneous distributed energy resources (DERs). This demands a distributed energy management (EM) to manage power generation and delivery of energy sources to maintain power quality under the impact of EV charging, to save operating costs, and to enhance resiliency. However, the global optimality of the distributed EM's optimization problem is still an issue in existing work because of the non-convex nature of the optimization problem. In this paper, a distributed EM strategy for grid-connected distribution networks is proposed. In particular, the EM strategy is composed of two steps. In the first step, some conditions of the EM optimization task are relaxed to apply an algorithm converging to the global optimality. The results of the first step are used to reconfigure constraints of the full optimization problem in Step 2. The proposed scheme is validated by implementing the real-time controller-hardware-in-the-loop (CHIL) experimentation on the IEEE 33 bus system. To study the impact of EV charging, EV data is collected from the market and the literature to generate realistic EV load profiles to demonstrate the effectiveness of the proposed strategy on saving operating costs and maintaining power quality.
\end{abstract}

Index Terms-Electric vehicles, distributed energy management, distribution grid, controller-hardware-in-the-loop, realtime simulation.

\section{NOMENCLATURE}

$n, m$

Bus index

$r, s$ Region index

$N, R$ Number of buses and regions, respectively.

${ }^{*}$ P. H. Hoang, G. Ozkan, P. R. Badr, and C. S. Edrington are with the Real-Time COntrol and Optimization Laboratory (RT-COOL)-Holcombe Department of Electrical and Computer Engineering-Clemson University, Clemson, SC 29634, USA. E-mails: $\{$ huuphuh,gokhano, prameza, cedring\}@clemson.edu.

$\dagger$ B. Papari is with Energy Production \& Infrastructure Center (EPIC), University of North Carolina, 8700 Phillips Rd, Charlotte, NC 28223, USA E-mail: bpapari@uncc.edu.

¥ M. A. Zehir and L. Mehigan are with Energy Policy and Modelling Group, MaREI Centre, Environmental Research Institute, University College Cork, Old Lee Road, Cork, Ireland. E-mails: \{alparslan.zehir,laura.mehigan\}@ucc.ie.

$\perp$ B. Hayes is with School of Engineering and Architecture, University College Cork, College Road, Cork, Ireland. E-mail: \{barry.hayes\}@ucc.ie.

$\S$ D. A. Kez is with School of Electronics, Electrical Engineering and Computer Science, Queen's University Belfast, Belfast, BT9 5AH, United Kingdom. E-mail: dalkez01@qub.ac.uk.

T A. M. Foley is with School of Mechanical and Aerospace Engineering and School of Electronics, Electrical Engineering and Computer Science, Queen's University Belfast, Belfast, BT9 5AH, United Kingdom. E-mail: a.foley@qub.ac.uk.

$\mathcal{N}, \mathcal{R}$
$\mathcal{R}_{r}$
$\mathcal{N}_{r}$
$M G, M T, P V$
$E V, L$
$\mathcal{M T}$
$\bar{a}, \underline{a}$
$V_{n}, V_{n}^{r e}, V_{n}^{i m}$
$V_{r, n}^{r e}, V_{r, n}^{i m}$
$P_{n}^{A}, Q_{n}^{A}$
$P_{r}^{D}$
$P_{n}^{S}$

Set of all buses and set of all regions in the network, respectively.

Set of buses in region $r$.

Set of neighboring regions of region $r$. Main grid, microturbine, and photovoltaic system, respectively.

Electric vehicle and non-EV load, respectively. Set of buses which contain a microturbine. Upper and lower limits of $a$, respectively. Complex bus voltage at bus $n$, its real part, and its imaginary part, respectively. Real part and imaginary part of bus voltage at bus $n$ optimized at region $r$, respectively. Active and reactive power generated, supplied, or consumed by $A$ at bus $n$, respectively. Dispatchable active power of region $r$. Sum of non-dispatchable active power and active load at bus $n$.

\section{INTRODUCTION}

$\mathbf{E}$ Vs are widely considered as a replacement for internal combustion engine vehicles because they are environmentally friendly and beneficial to sustainable energy goals. However, the increasing of EV penetration and EV charging capacities negatively impact distribution grids. For example, it is shown in [1] that a distribution grid fails to maintain required voltages when the EV penetration level reaches $30 \%$. Besides, it is expected that distribution grids will be populated with heterogeneous DERs [2]. Therefore, a distributed EM coordinating dispersed energy sources to gain economic objectives while maintaining power quality under the impact of $\mathrm{EV}$ penetration is valuable.

\section{A. Literature Review}

EM is one of the constituents of future distribution grids' control and management system [3]. The primary function of EM is to optimally allocate power generation and delivery to sources of energy in a well-defined sense while adhering to system constraints [4-10]. Distributed EM is considered superior to the centralized counterpart in terms of resiliency 
and scalability. Yet, the global optimality of the distributed EM's optimization problem is still an issue.

The alternating direction method of multipliers (ADMM) [11] is a popular method to solve the EM's optimization problem [10, 12-14]. The popularity of the ADMM is attributed to its efficiency and straightforwardness as it is applied to various applications with three clear updating steps. It is worth noting that the theoretical guarantee of convergence of the ADMM is confined to convex optimization problems. However, the EM's optimization problem for AC networks is known to be non-convex that the ADMM fails to deliver the convergence guarantee [10]. Recently, the authors in [15] apply the augmented Lagrangian alternating direction inexact Newton method to the EM's optimization problem. The method overcomes the convergence issue for non-convex problems, yet global optimality is still not ensured. In addition, each iteration of the method generally has higher complexity and computation than the ADMM. There are papers that do not consider the bus voltage constraints and power limits on transmission lines in the EM's optimization problem [16]. However, it is likely that these constraints are violated with the adoption of DERs and EVs.

Realistic representation of EV charging demand is needed to accurately examine the effectiveness of innovative EM approaches. The studies in the literature are mainly based on either direct use of charging profile data collected from field demonstrations [17] or deterministic charging scenarios built on general assumptions [18]. However, these conventional methodologies have some drawbacks. Charging data from a field pilot usually has limited representation (due to a low number of participants, short testing period, and cars from a few brands and constrained availability to other researchers. Furthermore, assumptions about using the same charging demand and energy storage capabilities, considering starting and ending times from a limited time period of the day, straightforward determination of the number of charging sessions, initial and final State of Charge (SoC) values, distinguished consideration of slow and fast charging profiles are considerably far from observations in the field [19].

For a better representation of the EV charging demand of a large group of customers, a novel and easily replicable methodology is developed and used in this paper. It is based on the combined use of characteristics of a wide range of cars available on a country level, charging starting and ending time probabilities, SoC value probabilities, and driving statistics. A unique feature of the methodology is consecutive relations built between slow and fast charging sessions.

\section{B. Statement of Contributions}

In this paper, a two-step distributed EM scheme is proposed. The first step is to search for the global optimum by relaxing some constraints in the EM's optimization problem to reduce it to a convex one. In doing so, an algorithm that secures globally optimal solutions can be applied. Its optimized results are used to reconfigure dispatchable active and reactive power constraints in the second step in which the full problem is considered. Reconfiguring the active power constraints makes an ADMM-based algorithm in the second step seeks solutions in a narrowed region around the global optimality. Besides, the reconfiguration of the reactive constraints ensures power factor (PF) requirements are satisfied. EV charging load profiles, generated through a novel approach for a realistic representation of daily cases in EV integrated distribution grids, are used to test the proposed strategy. Slow and fast charging sessions of a large number and a wide range of cars available in the US market are considered together in the explored scenarios. Based on a thorough literature review, there is no existing work implementing distributed EM via a CHIL setup. This work is one step further as a CHIL experiment is constructed and real-time experimental evidence is shown.

\section{Paper Organization}

The remainder of this paper is organized as follows. In Section II, the EM problem is derived and formulated. For ease of understanding, common notations are presented in the section. Section III is dedicated to present the two-step distributed EM strategy. The CHIL demonstration of the proposed scheme is discussed in Section IV. Section V concludes the paper and discusses future directions.

\section{Problem Formulation}

Consider a grid-connected radial distribution network consisting of heterogeneous DERs dispersed over the network: microturbine(s) (MT), wind turbine(s) (WT), and photovoltaic (PV) $\operatorname{system(s).~It~is~desired~that~the~grid~1)~operates~at~}$ economically optimal points, 2) maintains voltage satisfaction for all the buses, and 3) adheres to power generation and delivery capacity limits while meeting the power balance constraint.

\section{A. System Modeling and Notations}

Let an undirected graph $(\mathcal{N}, \mathcal{E})$ represent the grid, where $\mathcal{N}=\{1, \ldots, N\}$ is the set of nodes and $\mathcal{E}=\{(n, m)\} \subseteq$ $\mathcal{N} \times \mathcal{N}$ is the set of lines. The terms bus and node are used interchangeably in this paper. The bus connecting directly with the substation is indexed 1 . Let $\mathcal{M T} \subset \mathcal{N}$ be the set of nodes that have MTs installed. The system is divided into $R$ regions; each has a dispatchable source of energy, either a substation or an MT. Assume that each bus in a region does not have more than one connection with buses outside the region. Let $\mathcal{R}=\{1, \ldots, R\}$ and $\mathcal{R}_{r}$ be the set of nodes in region $r$. Denote $\mathcal{N}_{r}$ as the set of neighboring regions of region $r$.

Let $\operatorname{Tr}(),.(.)^{*},(.)^{T}$, and $(.)^{H}$ be the trace operator, complex conjugate, transposition, and complex conjugate transposition operators, respectively. Let $\left[a_{i j}\right]_{l \times k}$ be a $l \times k$ matrix. Throughout this paper, there are some common notations. $P$ and $Q$ accordingly indicate active and reactive power. Subscripts $m$ and $n$ are the bus index and subscripts $r$ and $s$ are the region index. Moreover, superscripts re, im, MG, MT, EV, $P V$, and $L$ accordingly indicate the real part, the imaginary part, the main grid, MT, EV, PV, and non-EV load. For example, with these notations, $P_{n}^{M T}$ and $Q_{n}^{M T}$ are active and reactive power generated from an MT at bus $n$, respectively. Overline 
and underline indicate lower and upper limits of a quantity, respectively. Let $V_{n}=V_{n}^{r e}+j V_{n}^{i m}$ be the bus voltage at bus $n$. The sign of active power $P_{n}$ (reactive power $Q_{n}$ ) at node $n$ is defined as follows: if $P_{n}>0\left(Q_{n}>0\right)$, then node $n$ injects active (reactive) power to the network, otherwise it absorbs active (reactive) power.

\section{B. Cost Functions}

The active power of the dispatchable sources can be adjusted to gain economic objectives formed in the following function:

$$
f_{1}\left(P_{1}^{M G}\right)+\sum_{n \in \mathcal{M} \mathcal{T}} f_{n}\left(P_{n}^{M T}\right)
$$

The first term in (1) is the cost of buying power from the main grid which is a multiplication of the market price $c^{M G}$ $(\$ / k W h)$ and the amount of active power supplied from the main grid. The second term is the cost of power generated by the MTs which has the following form

$$
f_{n}\left(P_{n}^{M T}\right)=c^{F}\left(\alpha_{n}\left(P_{n}^{M T}\right)^{2}+\beta_{n} P_{n}^{M T}+\gamma_{n}\right), \alpha_{n}>0
$$

where $c^{F}$ (\$/gallon) is the cost of fuel.

\section{EV Charging}

A probabilistic charging profile generation methodology that combines characteristics and statistics from a range of data resources is employed. The profile generator builds relations between slow and fast charging sessions to represent the EV charging profile of a large number of cars, including residential and public charging cases.

In the first stage, characteristics of the 23 cars that are available in the US market over the last decade are considered based on the information available in [20] and [21]. Slow and fast charging demand and time, energy storage capability and energy consumption per kilometer are imported into the charging profile generation methodology as the main car characteristics. The slow charging demand of the considered cars ranges between 3.6 to $16.5 \mathrm{~kW}$ with 3 to 14 hours full charging time. The fast charging demand of the considered EVs ranges between 22 to $110 \mathrm{~kW}$ with 20 to 80 minutes charging time up to around $90 \%$ SoC level. Depending on the number of customers defined in a scenario, a random EV from the determined pool of cars is assigned to each customer.

In the next stage, probabilities for slow charging sessions are defined based on [19]. The detailed probabilities are available in the cited publication. These consist of the overall share of customers who charge their car at least once a day, probabilities of charge starting time (in 15-minute ranges), initial and final SoC values (in $8.33 \%$ ranges -due to SoC recording resolution used in the considered field pilot-, depending on the period of starting time, called as morning peak between 06:00 AM and 09:00 AM, evening peak from 03:00 PM to 09:00 PM and the rest of the day). After a range is selected for a customer's charging session, a random number inside that range is chosen as the exact starting time. A similar approach is followed to assign initial and final $\mathrm{SoC}$ values for the considered sessions. Using the assigned initial and final SoC values and charging times from car characteristics, the charging duration for each car is calculated. Taking into account the assigned charge starting times and the calculated charging durations, charge ending times are derived. Additional idle waiting times/parking times are assigned based on the statistics provided in [22]. A charging model that is based on the constant current charging up to around $90 \%$ SoC, and then the linear limit of power for most of the vehicles provided in [23] is used as part of the profile generation tool. The details of this model can be found in the reference. A transitional period follows the slow charging session, considering only the cars that are charged more than once a day. During this period, cars are driven, spend the energy stored in their batteries, and park based on the comprehensive statistics provided in [24] on distance traveled and time spent between charging sessions.

The fourth stage is fast charging in public charging stations. Considering the departure times from the previous charging session and adding on the time spent during the transitional period, fast charge starting times are calculated. In a similar manner, considering the final SoC values from the previous charging sessions and the energy spent/SoC reduced (calculated according to the distance traveled and energy consumed per kilometer) between two charging sessions, the initial SoC values for the fast charging session are derived. Since full fast charging times (ranging from 20 to 80 minutes, with around 40 minutes on average) are below typical charging durations, all the cars are assumed to be fully charged up to $90 \%$ SoC in fast charging sessions. Considering the derived fast charge starting times, initial SoC values, and minimum charging up to $90 \%$ SoC, fast charge ending times are calculated. As in stage 2 , an additional time for parking or idle waiting is assigned, and departure times are calculated.

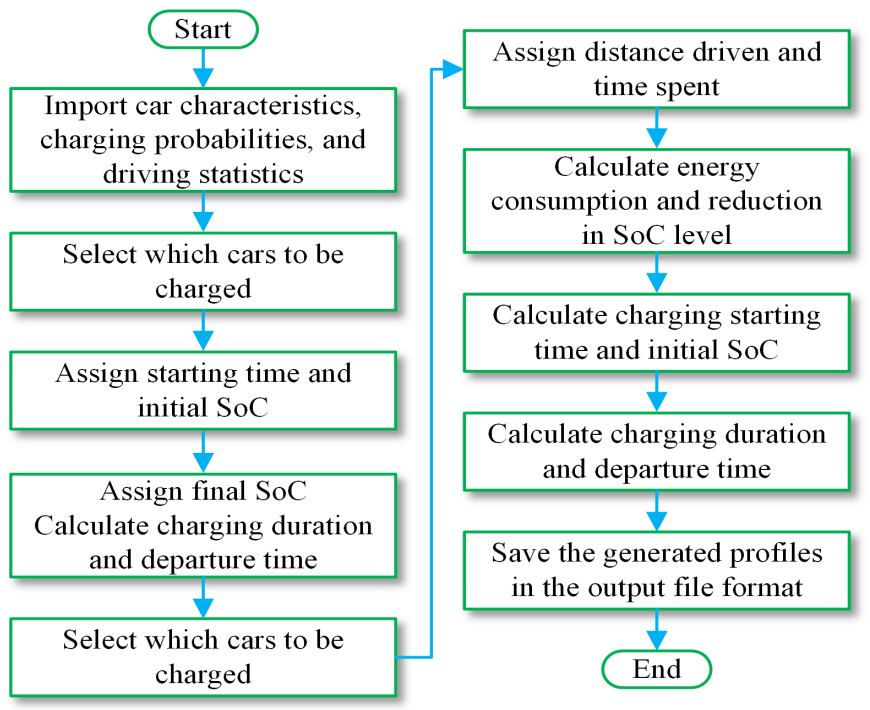

Fig. 1. EV load profile generation methodology.

If a charging session ends on the next day, all the hours with charging demand in the next day are also considered. This is required to make clear comparisons between the cases without and with distributed EM. Random assignment of cars to each customer and random selection of the exact charge 
starting times and exact initial $\mathrm{SoC}$ values for the first slow charging session allow generation of different individual and aggregated daily charging profiles at each run.

\section{Constraints}

An MT unit has lower and upper generation capacity limits on both active and reactive power as

$$
\begin{aligned}
& \underline{P_{n}^{M T}} \leq P_{n}^{M T}=\left(P_{n}-P_{n}^{L}-P_{n}^{E V}\right) \leq \overline{P_{n}^{M T}} \\
& \underline{Q_{n}^{M T}} \leq Q_{n}^{M T}=\left(Q_{n}-Q_{n}^{L}-Q_{n}^{E V}\right) \leq \overline{Q_{n}^{M T}}
\end{aligned}
$$

Similarly, the main grid is imposed these constraints as

$$
\begin{aligned}
& \underline{P_{1}^{M G}} \leq P_{1}^{M G}=\left(P_{1}-P_{1}^{L}-P_{1}^{E V}\right) \leq \overline{P_{1}^{M G}} \\
& \underline{Q_{1}^{M G}} \leq Q_{1}^{M G}=\left(Q_{1}-Q_{1}^{L}-Q_{1}^{E V}\right) \leq \overline{Q_{1}^{M G}}
\end{aligned}
$$

For a node that has a PV installed, the sum of all injections and withdrawals at the bus equal 0 ; that is,

$$
P_{n}-\left(P_{n}^{P V}+P_{n}^{L}+P_{n}^{E V}\right)=0
$$

It is assumed that PV systems actively participate in regulating bus voltages by adjusting reactive power, which is confined in following range:

$$
\underline{Q_{n}^{P V}} \leq Q_{n}^{P V}=\left(Q_{n}-Q_{n}^{L}-Q_{n}^{E V}\right) \leq \overline{Q_{n}^{P V}}
$$

WTs are similar to PVs as

$$
\begin{aligned}
& P_{n}-\left(P_{n}^{W T}+P_{n}^{L}+P_{n}^{E V}\right)=0 \\
& \underline{Q_{n}^{W T}} \leq Q_{n}^{W T}=\left(Q_{n}-Q_{n}^{L}-Q_{n}^{E V}\right) \leq \overline{Q_{n}^{W T}} .
\end{aligned}
$$

Let bus 1 be the slack bus. The voltage magnitude at bus $n \in \mathcal{N} \backslash\{1\}$ are desired to be confined in a bounded region defined as

$$
\underline{\left|V_{n}\right|} \leq\left|V_{n}\right| \leq \overline{\left|V_{n}\right|}
$$

Typically, $\left|V_{n}\right|=0.95$ per units $(\mathrm{pu})$ and $\overline{\left|V_{n}\right|}=1.05 \mathrm{pu}$ are used. If phase angles of voltages at the buses are confined, power transmitted on lines are bounded. Therefore, a constraint is set on the voltage phase angle at bus $n \in \mathcal{N} \backslash\{1\}$ as

$$
-\overline{\theta_{n}} \leq \theta_{n}=\arctan \left(\frac{V_{n}^{i m}}{V_{n}^{r e}}\right) \leq \overline{\theta_{n}}
$$

\section{E. EM's Optimization Task}

Given the costs and constraints presented in the previous subsections, the optimization task is stated as

$$
\begin{aligned}
& \quad \min \left(f_{1}\left(P_{1}^{M G}\right)+\sum_{n \in \mathcal{M} \mathcal{T}} f_{n}\left(P_{n}^{M T}\right)\right) \\
& \text { s.t. } \quad(3),(4),(5),(6),(7),(8),(9) .
\end{aligned}
$$

The problem (10) can be transformed into an equivalent one which has voltages as the only variables [25, 26]. Denote the bus voltage vector by $\mathbf{V}=\left[V_{1}, \ldots, V_{N}\right]^{T}$ and the bus admittance matrix by $\mathbf{Y}=\left[Y_{l k}\right]_{N \times N}$. Let $\left\{\mathbf{e}_{n}\right\}_{n \in \mathcal{N}}$ form standard basis vectors in $\mathbb{R}^{n}$, in which $\mathbf{e}_{n}:=[0, \ldots, 1, \ldots, 0]^{T}$ is defined as the $n$-element of $\mathbf{e}_{n}$ is 1 and the others are 0 . The following transformation can be obtained $[25,26]$ :

$$
\begin{aligned}
& \mathbf{Y}_{n}:=\mathbf{e}_{n} \mathbf{e}_{n}^{T} \mathbf{Y} \\
& \left|V_{n}\right|^{2}=\operatorname{Tr}\left(\mathbf{V} \mathbf{V}^{H}\right) \\
& \mathscr{P}_{n}:=\frac{1}{2}\left(\mathbf{Y}_{n}+\left(\mathbf{Y}_{n}\right)^{H}\right) \\
& P_{n}=\operatorname{Tr}\left(\mathscr{P}_{n} \mathbf{V} \mathbf{V}^{H}\right) \\
& \mathscr{Q}_{n}:=\frac{j}{2}\left(\mathbf{Y}_{n}-\left(\mathbf{Y}_{n}\right)^{H}\right) \\
& Q_{n}=\operatorname{Tr}\left(\mathscr{Q}_{n} \mathbf{V} \mathbf{V}^{H}\right)
\end{aligned}
$$

$P_{n}$ and $Q_{n}$ can be expressed as functions of $\mathbf{V}$, so do the cost function and the constraints in (10). It is well known that the optimization problem in (10) is non-convex [10]. In the following, a two-step distributed strategy is proposed to solve the non-convex optimization problem.

\section{Distributed EM Strategy}

In this section, a distributed strategy, which consists of two steps, is introduced and discussed. In the first step, the optimization task is relaxed by neglecting the voltage and reactive power constraints; i.e., (10) is relaxed to

$$
\begin{aligned}
& \quad \min f\left(\mathbf{P}^{D}\right)=\left(f_{1}\left(P_{1}^{M G}\right)+\sum_{n \in \mathcal{M} \mathcal{T}} f_{n}\left(P_{n}^{M T}\right)\right) \\
& \text { s.t. } \quad(3 a),(4 a),(5),(7 a) .
\end{aligned}
$$

where $\mathbf{P}^{D}=\left[\ldots, P_{r}^{D}, \ldots\right]^{T}, r \in \mathcal{R}$, is a vector consisting of the dispatchable active power of all the regions, and $P_{r}^{D}$ is $P_{1}^{M G}$ if region $r$ has the substation and $P_{n}^{M T}$ if region $r$ has an MT at bus $n$.

The goal of the first step is to find the optimal allocation of active power for the dispatchable sources. Its optimized outcomes are then fed into the second step in which constraints (3) and (4) are reconfigured based on the inputs. The active power inequality constraints are reconfigured such that the box constraints are narrowed to the region around the solutions of (12), which are denoted as $P^{M G, *}$ and $P_{n}^{M T, *}$, as

$$
\begin{aligned}
& \max \left(P_{n}^{M T, *}-\Delta P_{n}^{M T}, P_{n}^{M T}\right) \leq P_{n}^{M T} \leq \\
& \min \left(P_{n}^{M T}, *+\Delta P_{1}^{M T}{ }_{n}, \overline{P_{n}^{M T}}\right) \\
& \max \left(P^{M G, *}-\Delta P_{1}^{M G}, \underline{P^{M G}}\right) \leq P_{1}^{M G} \leq \\
& \min \left(P_{1}^{M G, *}+\Delta P_{1}^{M G}, \overline{P_{1}^{M G}}\right)
\end{aligned}
$$

where $\Delta P_{n}^{M T}=\sigma\left|P_{n}^{M T, *}\right|$ and $\Delta P_{1}^{M G}=\sigma\left|P_{1}^{M G, *}\right|$, where $0<\sigma<1$, and $\sigma=0.1$ is selected in this paper. For the reactive power inequality constraints at buses where MTs are installed, a minimum PF of 0.95 with active power referred to solutions of (12) are allowed. In the second step, the optimization task (10) is considered. In [16], only Step 1 is considered. However, with the adoptions of EVs and DERs, the voltage constraints and power transmission limits on transmission lines are likely violated. There are works that consider only Step 2 and utilize the ADMM to solve the EM's optimization problem [12-14]. But (10) is a nonconvex optimization problem, and the convergence and global optimality are not ensured. 


\section{A. Step 1}

Denote the sum of non-dispatchable active power and loads at bus $n$ by $P_{n}^{S}=P_{n}^{P V}+P_{n}^{W T}+P_{n}^{L}+P_{n}^{E V}, \forall n \in \mathcal{N}$. The augmented Lagrangian of (12) is

$$
\begin{aligned}
L_{1} & =f\left(\mathbf{P}^{D}\right)+\mu\left(\sum_{r \in \mathcal{R}} P_{r}^{D}+\sum_{n \in \mathcal{N}} P_{n}^{S}\right) \\
& +\sum_{r \in \mathcal{R}} \lambda_{r}^{\max }\left(P_{r}^{D}-\overline{P_{r}^{D}}\right)+\sum_{r \in \mathcal{R}} \lambda_{r}^{\min }\left(-P_{r}^{D}+\underline{P_{r}^{D}}\right) \\
& +\frac{\rho_{1}}{2}\left\|\sum_{r \in \mathcal{R}} P_{r}^{D}+\sum_{n \in \mathcal{N}} P_{n}^{S}\right\|^{2}
\end{aligned}
$$

where $\mu, \lambda_{r}^{\max }$, and $\lambda_{r}^{\min }$ are Lagrange multipliers, and $\rho_{1}>0$. In [27] and [28], a distributed algorithm based on the singular perturbation method is proposed aiming to solve a general optimization problem. The underlying idea of the algorithm is constructing a two-time-scale dynamical system which has an equilibrium point satisfying the Karush-Kuhn-Tucker conditions: the first set of equations is called the fast dynamic layer and the second set is called the slow dynamic layer. The fast dynamic layer can be derived as

$$
\begin{aligned}
\dot{\xi}_{r}^{h}= & -\xi_{r}^{h}-\sum_{s \in \mathcal{N}_{r}}\left(\xi_{r}^{h}-\xi_{s}^{h}\right)-\sum_{s \in \mathcal{N}_{r}}\left(\zeta_{r}^{h}-\zeta_{s}^{h}\right) \\
& +\left(P_{r}^{D}+\sum_{n \in \mathcal{R}_{r}} P_{n}^{S}\right) \\
\dot{\zeta}_{r}^{h}= & \sum_{s \in \mathcal{N}_{r}}\left(\xi_{r}^{h}-\xi_{s}^{h}\right) \\
\dot{\xi}_{r}^{\mu}= & -\xi_{r}^{\mu}-\sum_{s \in \mathcal{N}_{r}}\left(\xi_{r}^{\mu}-\xi_{s}^{\mu}\right)-\sum_{s \in \mathcal{N}_{r}}\left(\zeta_{r}^{\mu}-\zeta_{s}^{\mu}\right)+\mu_{r}, \\
\dot{\zeta}_{r}^{\mu}= & \sum_{s \in \mathcal{N}_{r}}\left(\xi_{r}^{\mu}-\xi_{s}^{\mu}\right)
\end{aligned}
$$

It is noted that (15a) is to estimate the average of $\left(\sum_{r \in \mathcal{R}} P_{r}^{D}+\right.$ $\left.\sum_{n \in \mathcal{N}} P_{n}^{S}\right)$, i.e., $\xi_{r}^{h} \longrightarrow \frac{1}{R}\left(\sum_{r \in \mathcal{R}} P_{r}^{D}+\sum_{n \in \mathcal{N}} P_{n}^{S}\right)$. The following is the slow dynamic layer

$$
\begin{aligned}
& \dot{x}_{r}=-\epsilon k_{r}^{x}\left(\frac{\partial f_{r}}{\partial x_{r}}\left(x_{r}\right)+\xi_{r}^{\mu}+\lambda_{r}^{\max }-\lambda_{r}^{\text {min }}+\rho_{1} \xi_{r}^{h}\right) \\
& \dot{\mu}_{r}=\epsilon k_{r}^{\mu}\left(\xi_{r}^{h}-\sum_{s \in \mathcal{N}_{r}}\left(\mu_{r}-\mu_{s}\right)\right) \\
& \dot{\lambda}_{r}^{\max }=\epsilon k_{r}^{\lambda^{\max }}\left(x_{r}-\overline{P_{r}^{D}}\right) \\
& \dot{\lambda}_{r}^{\text {min }}=\epsilon k_{r}^{\lambda^{\text {min }}}\left(-x_{r}+\underline{P_{r}^{D}}\right)
\end{aligned}
$$

While $\epsilon \ll 1$ is a real positive number which aims to have (16) having a slower dynamics than that of (15), $k_{r}^{x}, k_{r}^{\mu}, k_{r}^{\lambda^{\max }}$, and $k_{r}^{\lambda^{\text {min }}} \in \mathbb{R}_{>0}$ are to cope with differences in the dynamics of $x_{r}, \mu_{r}, \lambda_{r}^{\max }$, and $\lambda_{r}^{\min }$.

\section{B. Step 2}

The ADMM algorithm [11] is briefly presented before deriving its application to the considered optimization problem. Consider the following optimization problem:

$$
\begin{array}{ll} 
& \min (f(\mathbf{x})+g(\mathbf{z})) \\
\text { s.t. } & \mathbf{A} \mathbf{x}+\mathbf{B} \mathbf{z}=\mathbf{c} \text { and } \mathbf{x} \in \mathcal{C}_{x}, \mathbf{z} \in \mathcal{C}_{z}
\end{array}
$$

where $\mathbf{x}$ and $\mathbf{z}$ are vectors of variables, $\mathbf{c}$ is a constant vector, $\mathcal{C}_{x}$ and $\mathcal{C}_{z}$ are their constraint sets, and $\mathbf{A}$ and $\mathbf{C}$ are matrices. Suppose the vectors and matrices have appropriate dimensions. The associated augmented Lagrangian is

$$
\begin{aligned}
L_{2}(\mathbf{x}, \mathbf{y}, \mathbf{z})= & f(\mathbf{x})+g(\mathbf{z})+\mathbf{y}^{T}(\mathbf{A} \mathbf{x}+\mathbf{B} \mathbf{z}-\mathbf{c}) \\
& +\frac{\rho_{2}}{2}\|\mathbf{A} \mathbf{x}+\mathbf{B} \mathbf{z}-\mathbf{c}\|_{2}^{2}
\end{aligned}
$$

where $\rho_{2}>0$. The ADMM has three repetitively updating steps as

$$
\begin{aligned}
& \mathbf{x}^{k+1}=\underset{\mathbf{x} \in \mathcal{C}_{x}}{\operatorname{argmin}} L_{2}\left(\mathbf{x}, \mathbf{z}^{k}, \mathbf{y}^{k}\right) \\
& \mathbf{z}^{k+1}=\underset{\mathbf{z} \in \mathcal{C}_{z}}{\operatorname{argmin}} L_{2}\left(\mathbf{x}^{k+1}, \mathbf{z}, \mathbf{y}^{k}\right) \\
& \mathbf{y}^{k+1}=\mathbf{y}^{k}+\rho_{2}\left(\mathbf{A} \mathbf{x}^{k+1}+\mathbf{B} \mathbf{z}^{k+1}-\mathbf{c}\right)
\end{aligned}
$$

The ADMM's configuration is now specified to fit the optimization problem. Applying the ADMM for consensus optimization problem [11], let $g(\mathbf{z})=0$ and $\mathbf{c}=0$. Define a voltage vector $\mathbf{V}_{r}=\left[\ldots, V_{r, n}^{r e}, \ldots, V_{r, m}^{r e}, \ldots, V_{r, n}^{i m}, \ldots, V_{r, m}^{i m}, \ldots\right]^{T}$, where bus $n$ is in region $r$ and bus $m$ is not region $r$ but it has a neighbor in region $r$. Let $\mathbf{x}=\left[\mathbf{V}_{1}^{T}, \ldots, \mathbf{V}_{R}^{T}\right]^{T}$ and decompose $f(\mathbf{x})$ as

$$
f(\mathbf{x}) \equiv \sum_{n \in \mathcal{M} \mathcal{T} \cup\{1\}, n \in \mathcal{R}_{r}} f_{n}\left(\mathbf{V}_{r}\right)
$$

Define the matrix $\mathbf{A}=\left[a_{i j}\right]_{4 l \times k}$ such that it is full row rank and $l$ is the number of edges connecting two nodes of two different regions and $k$ is the dimension of $\mathbf{x}$. Additionally, $a_{i j}=|\kappa|$ if $j$-th element of $\mathbf{x}$, which is either $V_{r, n}^{r e}$ or $V_{r, n}^{i m}$ with bus $n$ has a neighbor not in region $r$. Otherwise, $a_{i j}=0$. The matrix $\mathbf{B}$ is defined as $\mathbf{B}=-\mathbf{I}_{4 l \times 4 l}$, where $\mathbf{I}_{4 l \times 4 l}$ is the $4 l \times 4 l$ identity matrix. In the second updating step, $z^{k+1}$ is the solution of the following equation

$$
\left.\frac{\Delta L_{2}\left(\mathbf{x}^{k+1}, \mathbf{y}^{k}, \mathbf{z}\right)}{\Delta \mathbf{z}}\right|_{\mathbf{z} \in \mathcal{C}_{z}}=0
$$

which can be expressed as

$$
\left.\mathbf{z}^{k+1}\right|_{\mathbf{z} \in \mathcal{C}_{z}}=\mathbb{P}\left(\frac{1}{\rho_{2}} \mathbf{y}^{k}+\mathbf{A} \mathbf{x}^{k+1}\right)
$$

If $\mathbf{y}$ has a zero initialization and $\mathbf{z}^{0}=\mathbb{P}\left(\mathbf{A} \mathbf{x}^{0}\right)$, then $\mathbf{z}^{k+1}=$ $\mathbb{P}\left(\mathbf{A} \mathbf{x}^{k+1}\right)$, where $\mathbb{P}$ is the orthogonal projection operator on $\mathcal{C}_{z}$. The orthogonal projection matrix $\mathbb{P}=\left[p_{i j}\right]_{4 l \times 4 l}$ is selected such that $p_{i i}=\frac{1}{2}, p_{i j}=-\frac{1}{2}$ if both the $i-$ th and $j-$ th elements of $\mathbf{A} \mathbf{x}^{k+1}$ are either the real part or the imaginary part of a bus voltage optimized in two regions, otherwise $p_{i j}=$ 0 . With the specified configuration, voltages of a bus (both the real part and imaginary) optimized in two different controllers reach a consensus as $\left(\mathbf{A} \mathbf{x}^{k}-\mathbf{z}^{k}\right) \longrightarrow 0$ when $k \longrightarrow \infty$.

\section{CHIL IMPLEMENTATION}

\section{A. CHIL Experimental Setup}

1) CHIL Setup: The IEEE 33 bus system in [29] with additions of DERs is used to validate the proposed scheme. The system is geographically divided into 6 regions, with each corresponding to an EM agent. These DERs' locations, parameters, and block diagrams are shown in Fig. 2a). Particularly, Rowen's model is used to simulate MTs' engines [30], and the 
1

2

3

4

5

6

7

8

9

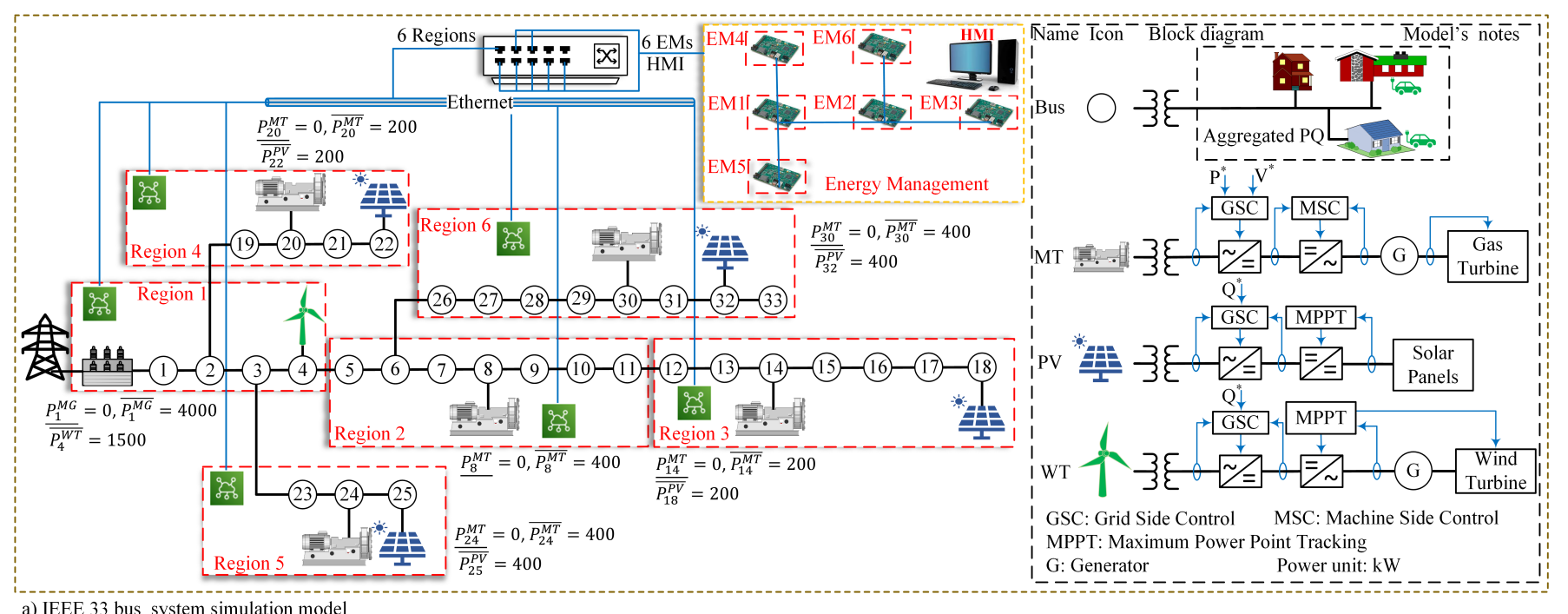

a) IEEE 33 bus system simulation model
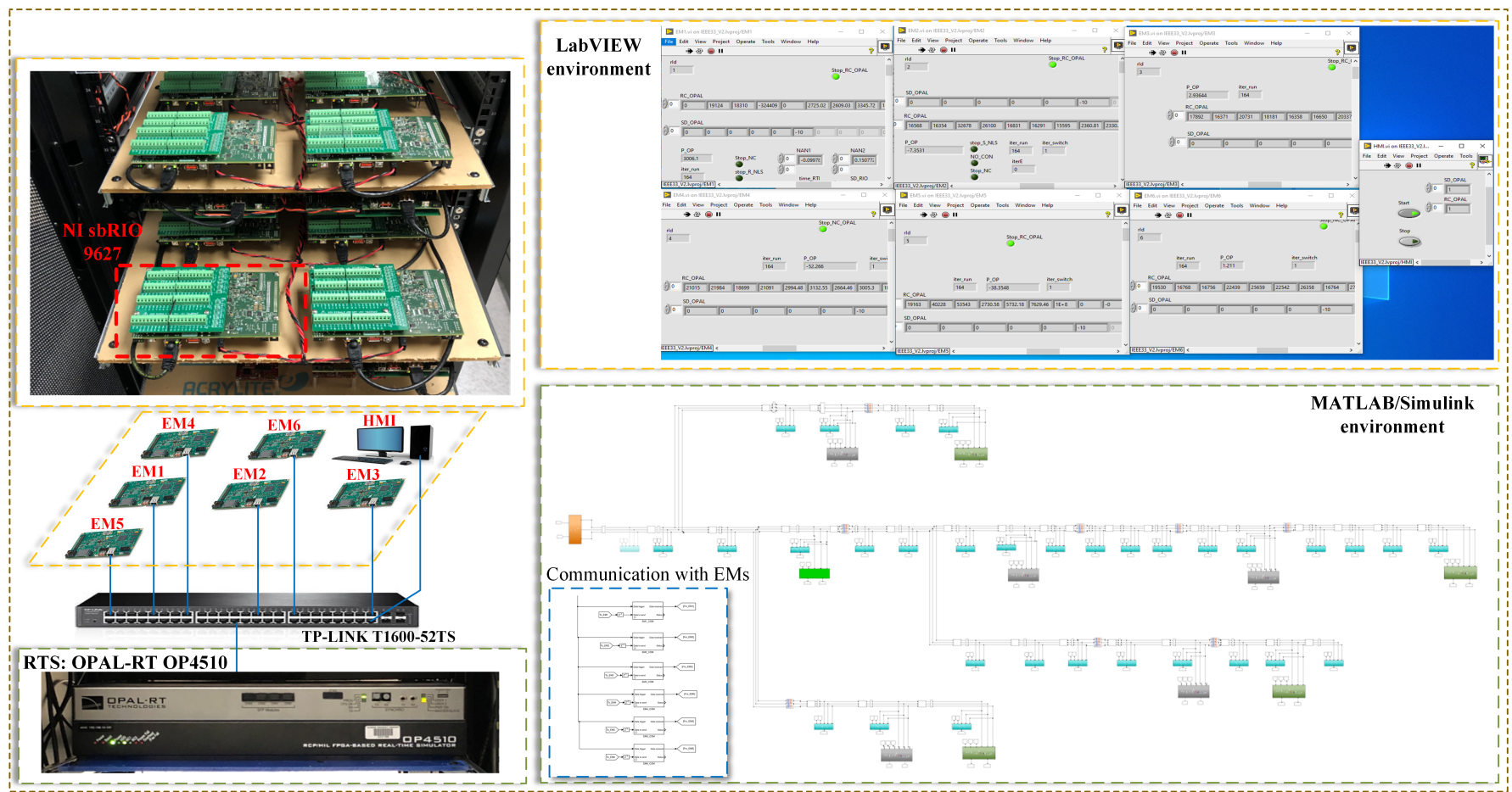

b) CHIL experimental setup

Fig. 2. CHIL experimentation: a) IEEE bus system simulation model b) CHIL experimental setup.

control structure in [31] is applied to integrate the MTs with the grid through power electronics converters. Furthermore, the type 4 wind turbine model in [32] is used, and the control model of the PVs is taken from [33]. Additionally, PV panels are modeled by the double-diode model with parameters extracted from the Siemens SM50 solar panel's specifications. Because the main focus of this paper is EM, average models for power electronics converters of the MTs, and PVs, and WT are sufficient to demonstrate the EM strategy.

Fig. 2b) illustrates the CHIL experimental setup. The system is simulated by MATLAB/Simulink and deployed into a digital real-time simulator (DRTS) OPAL-RT OP4510. The EM strategy is realized by LabVIEW and deployed into NI sbRIO 9627 controllers. A human-machine interface (HMI) computer is also added to the testbed for sending starting and stopping signals and collecting experimental data from the controllers. Experimental data from the model running in the DRTS is collected by OPAL-RT's OpWriteFile modules. A 1-Gbps ethernet network connecting these devices is established by a TP-LINK T1600G-52TS switch. The DRTS communicates with the controllers by the User Datagram Protocol (UDP), while the communication protocol among the controllers is the RTI Data Distribution Service (DDS). Moreover, the HMI exchanges data with the controllers by shared variables in LabVIEW. EM's results are passed to the model running in the DRTS as setpoints. The MTs have a duty of controlling active and reactive power injected into the network and bus voltages at which the MTs are installed, while the WT and the PVs are 
controlled to reach the commanded reactive power from EMs.

Fig. 3a) illustrates the EM program with a detail of the use of LabVIEW's Timed Loop and While Loop blocks. First, the program starts in a Timed Loop to initialize variables and buffers and to listen to starting signals from the HMI. After receiving the starting signal, there are two main blocks running in parallel: Optimization Block and Communication Block. The former is to implement the two-step optimization process while the latter exchanges data among the controllers and the simulator with the controllers. NI sbRIO 9627 boards are powered by a dual-core ARM Cortex-A9 microprocessor which allows the parallel running of the two blocks. Every 15 minutes, information of non-EV load, EV load, and weather data are fed into EMs; each EM can only receive this information of buses which it manages. Although these values can be generated from forecasting engines, they are stored in the DRTS and are sent to the controllers by the UDP protocol as this work primarily studies EM. Each controller uses this information and local system data of the region to form a LabVIEW cluster called sysInfo. Local system data includes the local admittance matrix and the WT and PVs' system parameters to estimate their power generation outputs given weather data. The information of cost functions and constraints is also in sysinfo.

A synchronous updating mechanism is designed in this work. In particular, the iteration index $k$ is added at the end of the exchanging data frame. A circular buffer reads the data coming from neighboring EMs, and the buffer is updated by the first-in-first-out rule if it discerns a new iteration index; otherwise, it ignores the receiving data. Every iteration, the controller reads the circular buffer and matches the iteration index in order to have the same $k$ for all involved EMs. This applies to both Step 1 and Step 2 as both steps use only the same circular buffer and RTI DDS's writer and reader block.

As an illustration of exchanging data between two EMs, Fig. $3 b)$ shows the exchange of data between EM1 and EM5. Buses that are managed in Region 1 are 1, 2, 3, and 4, and buses that are managed in Region 5 are 23, 24, and 25. These buses are illustrated by circles. There is a connection between nodes 3 and 23. Although Region 1 does not contain bus 23, EM1 has $V_{1,23}^{r e}$ and $V_{1,23}^{i m}$ as variables in the optimization process. Note that the first subscript indicates the region index, and the second indicates the bus index. Bus 23 in Region 1 is illustrated by a pentagon. Region 5 contains bus 23 and its bus voltage can be represented by $\left[V_{5,23}^{r e}, V_{5,23}^{i m}\right]$. The optimization process aims to reach a consensus between $\left[V_{1,23}^{r e}, V_{1,23}^{\text {im }}\right]$ and $\left[V_{5,23}^{r e}, V_{5,23}^{i m}\right]$. This applies to the other buses which have connections between two regions.

After Step 1, active and reactive power constraints are reconfigured by modifying corresponding fields in sysInfo, and then fed into Step 2. To solve (19a), the LabVIEW's Constrained Nonlinear Optimization module, which utilizes the sequential quadratic programming algorithm, is used. To obtain the information of the optimization problem, this module accesses sysInfo via a Formula Node with C++ syntax structure code deployed inside. Although it is observed that each iteration of Step 1 has a computational time fewer than $60 \mathrm{~ms}$ by implementing on the NI sbRIO 9627 controllers,

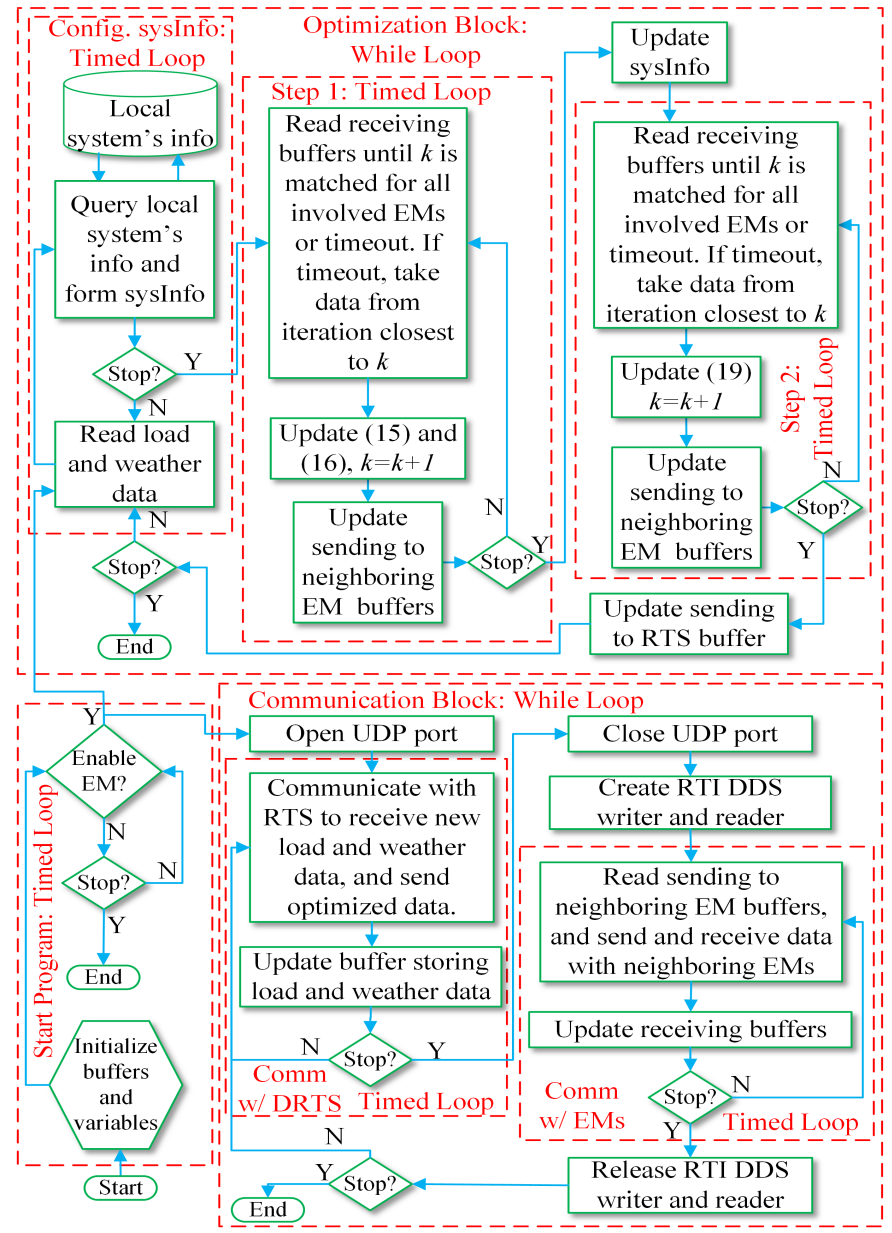

a) Diagram of EM's L $\bar{L} \bar{a} \bar{V} I \bar{E} \bar{W}$ program

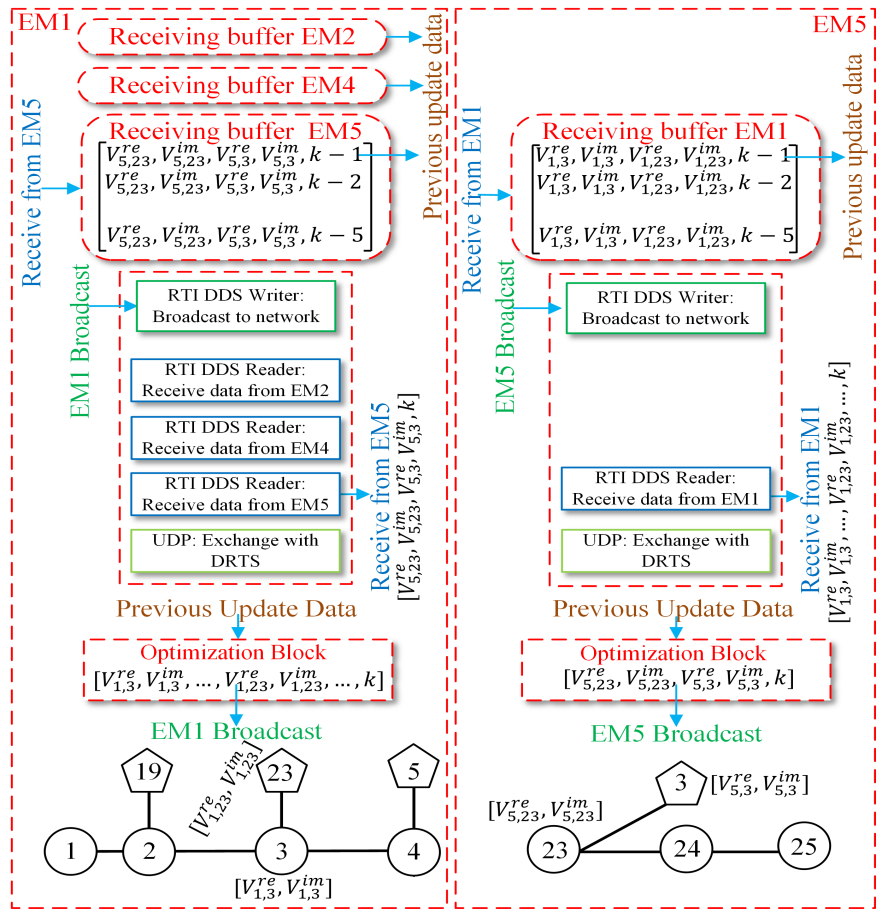

b) Illustration of exchanging data between EM1 and EM 5

Fig. 3. Distributed EM: a) Diagram of EM's LabVIEW program b) Illustration of exchanging data between EM1 and EM5. 
1

2

3

4

5

6

7

8

9 the time for each iteration of Step 1 is set to $60 \mathrm{~ms}$, which is higher than $40 \mathrm{~ms}$ of the Comm. w. EMs block in Fig. 3a) to ensure the communication time is less than the computational time of Step 1. Step 2 is more computationally expensive than Step 1 because of the nonlinear constraint solver; therefore, a duration of 3 seconds is set for each iteration. The maximum number of iterations is the stopping condition for both Step 1 and Step 2. The stopping conditions of the Comm w/ DRTS block and the Comm w/ EMs block are designed such that either Step 1 or Step 2 runs in parallel with the Comm w/ EMs block.
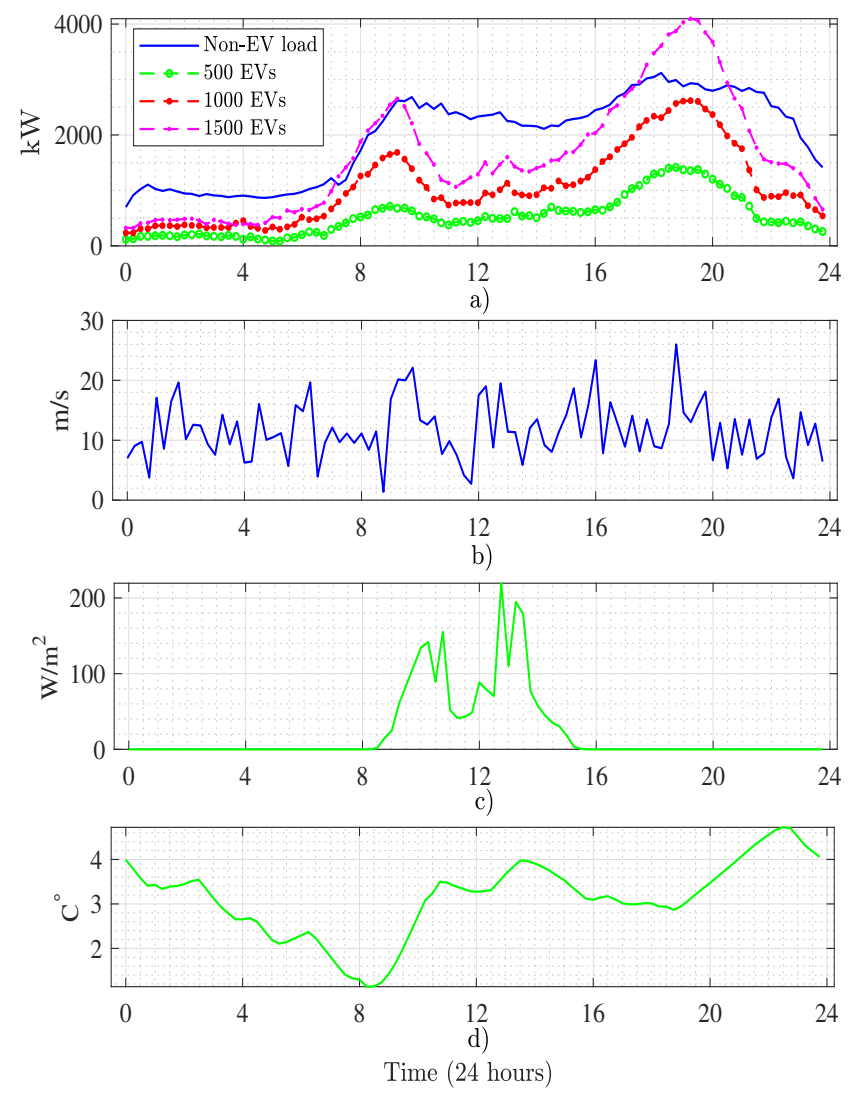

Fig. 4. Simulated operational data used in the experiment: a) load profile and renewable generation, b) wind speed, c) solar irradiance, and d) temperature.

2) Operational Data: The original load is replaced by the sum of EV load profiles generated from the method presented in Subsection II-C and non-EV load profiles generated from the CREST model [34]. 100 non-EV load profiles are generated from the model, and they are averaged. It is observed that the peak value of the 15-min averaged load per individual profile is around $1 \mathrm{~kW}$. Originally, the IEEE 33 bus system has a fixed 3.715 MW of load in total. Assuming the original load as the peak total demand and 3715 households in the residential area, load profiles are generated for each household. The load profiles are randomly distributed to the buses such that the number of load profiles is proportional to the original load values at the buses. The CREST model does not consider reactive power, so it is assumed PF follows uniform distribution in the range [0.95,0.99], which is close to that of [35]. For EV reactive load, a PF of 0.99 is assumed which is specified in Texas Instrument's design recommendation [36]. With the assumption of 3715 households, three levels of EV penetration are considered: $500 \mathrm{EVs}, 1000 \mathrm{EVs}$, and 1500 EVs. The number of EVs assigned to each bus is also proportional to the value of the original load. The fast-charging profile generation feature of the EV profile generation tool for the public charging stations outside the analysis area is taken into account to better represent the car arrival times and starting SoC levels of the second charging sessions for the residential chargers in the considered network. While the CREST model can be used to generate weather data for PVs, wind speed generation is not included in the model; therefore, it is generated by the Weibull distribution. Fig. 4 shows data generated by using the methodology described above for the day of January 15 . Furthermore, the market price of electricity is taken from [37], and the fuel price is $c^{F}=5.807$ ( $\$ /$ gallon) which is taken from [38] at the time of the experiment. The values of $\alpha_{n}, \beta_{n}$, and $\gamma_{n}$ of MTs at Buses 8,24 , and 30 are 0.12, 3.507, and 149.977, respectively. Those of MTs at Buses 14 and 20 are 0.097, 4.269, and 92.293, respectively. Additionally, the the market price of electricity is taken from [37].

\section{B. CHIL Experimental Results and Discussion}

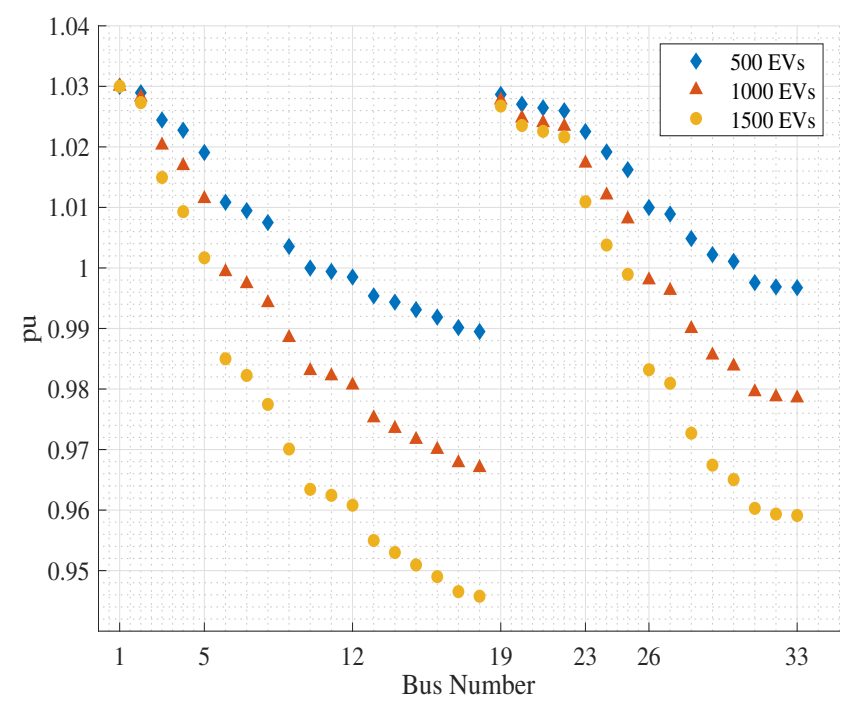

Fig. 5. Voltage profile of Scenario 1.

1) Scenario 1: The system without distributed EM is set up. MTs are simulated in the grid following mode that they generate active power to serve the load locally in their regions until they reach limits. The reactive power of the three types of DERs is set to 0 . With this setup, the impact of EV charging under the three levels of EV penetration at the peak load, which is at 19:30, is studied. Fig. 5 shows bus voltages of the three testing cases. As can be seen, while voltages for the cases of 500 and $1000 \mathrm{EVs}$ are in the range $[0.95,1.05] \mathrm{pu}$, buses 16,17 , and 18 have voltages less than 0.95 pu when the system populates $1500 \mathrm{EVs}$.

2) Scenario 2: This scenario follows the setup described in IV-A1 with 1500 EVs populated, and EMs are deployed. Three 

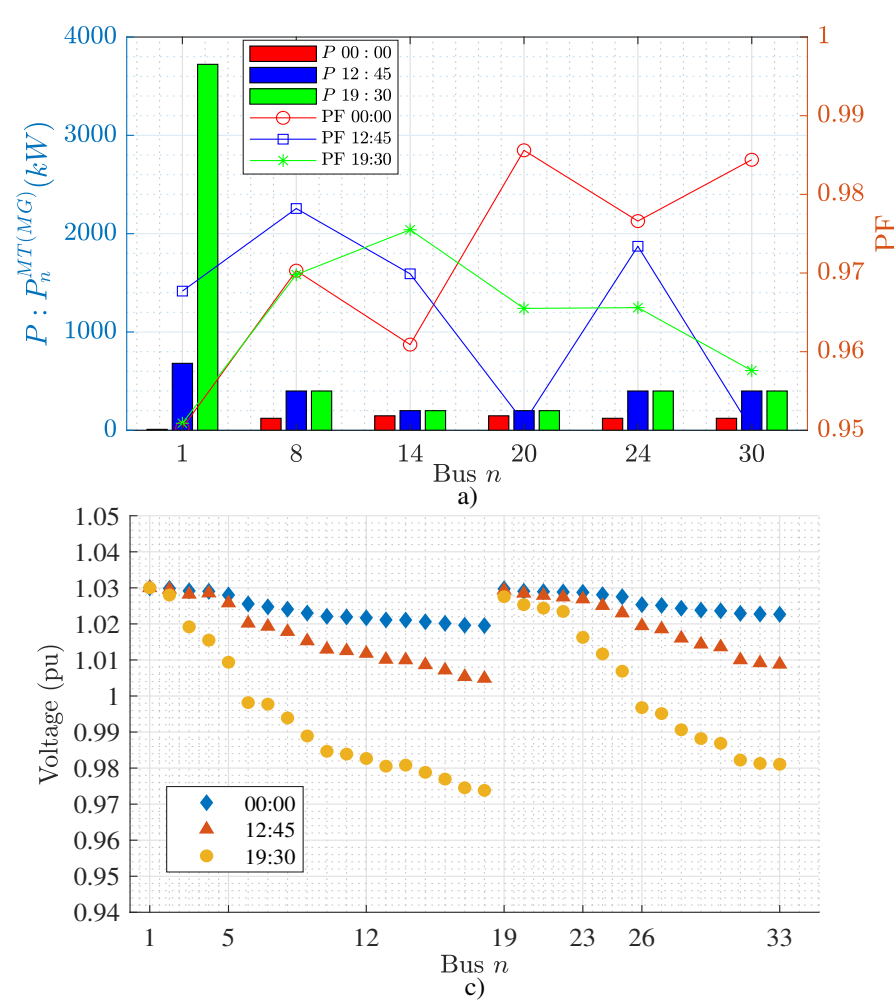
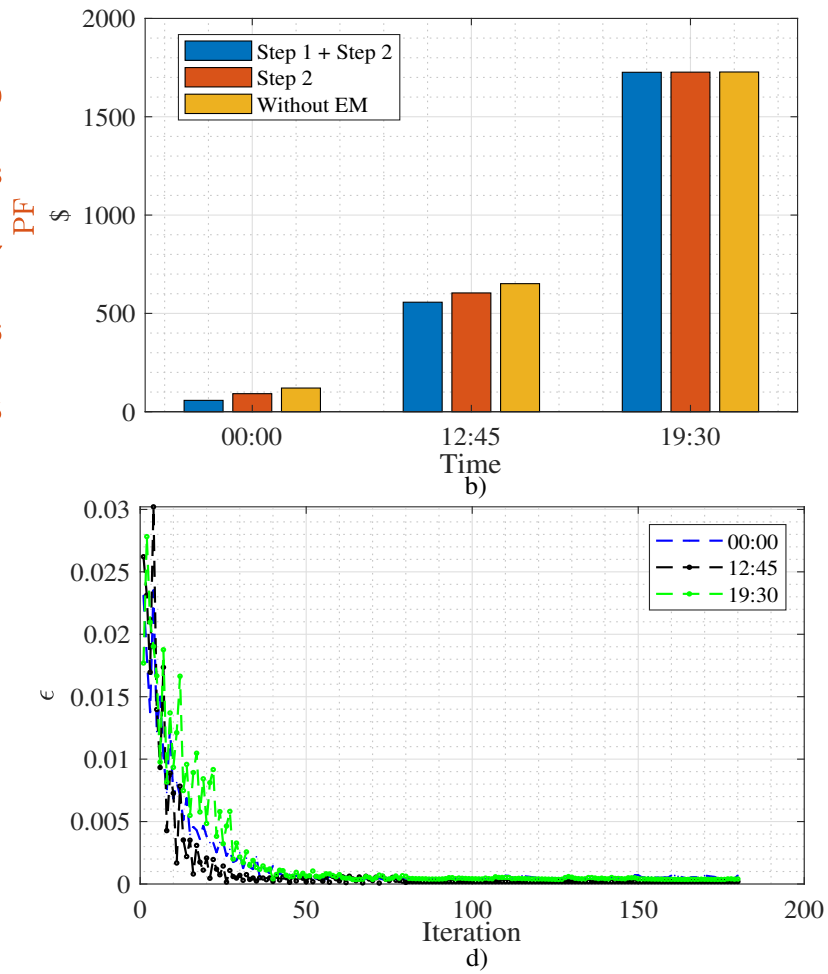

Fig. 6. Experimental results of Scenario 2: a) power generation allocation b) cost of generation c) bus voltages d) $\epsilon$.

cases are tested: at 00:00 when the load is the lowest, at 12:45 when the renewable generation is $\max$, and at 19:30 when the total load is at peak. The simulation results are shown in Fig. 6. Particularly, Fig. 6a) shows the amount of power generated or delivered at the 6 dispatchable power suppliers. For the case of 19:30, the MTs generate at full capacity. This is because the grid's electricity price is more expensive than the price if power generated by the MTs; therefore, EMs allocates power generation to the 5 MTs to meet the power load balance. The total cost of generation is shown in Fig. 6b) in which the case that generation allocated to the 6 energy sources proportionally to their maximum capacities is also computed; this case is without EM. It can be seen that deploying EM with both Step 1 and Step 2 is economically beneficial in the 12:00 AM and 12:45 PM cases. For the case of applying only Step 2, only local optimality is found. In Fig. 6c), voltages are in the range of $[0.95,1.05] \mathrm{pu}$. Compared to the case without EM, bus voltages are maintained under the impact of 1500 EVs. Define the following quantity

$$
\epsilon=\max _{n \in \Omega}\left|V_{r, n}-V_{s, n}\right|
$$

where $\Omega$ is the set of buses which have voltage optimized in both region $r$ and region $s . \epsilon$ is shown in Fig. 6d) and it can be seen that voltages converge after 60 iterations for all three cases.

\section{CONCLUSion AND Future Work}

In this paper, a distributed EM to efficiently manage distribution grids with the penetration of heterogeneous DERs and EV is presented. The strategy includes two steps that the first step enhances the global optimality searching for the second step. In addition, information after running Step 1 is used to reconfigure constraints to ensure that the PF limits of generators and the substation are respected. A realistic EV charging profile generation methodology is also presented in this work. The IEEE 33 bus system is used to investigate the impact of EVs. The CHIL implementation of the strategy is also reported, and the experimental evidence is shown. Experimental results show the economic and voltage regulation benefits of deploying the distributed EM.

There are potential directions to leverage this work in the future. First, energy storage technologies can be added to the current version. Second, adopting the vehicle-to-grid (V2G) mode into the system is another extension. Third, the future work will consider adding distribution locational marginal pricing of the power grid and the traffic flow of the transportation systems to the model.

\section{REFERENCES}

[1] G. A. Putrus, P. Suwanapingkarl, D. Johnston, E. C. Bentley, and M. Narayana. "Impact of electric vehicles on power distribution networks". 2009 IEEE Vehicle Power and Propulsion Conference, pp. 827-831.

[2] M. Henderson, D. Novosel, and M. L. Crow. "Electric power grid modernization trends, challenges, and opportunities". 2017.

[3] B. P. Hayes, M. Prodanovic. "State forecasting and operational planning for distribution network energy management systems". IEEE Transactions on Smart Grid, vol. 7, no. 2 (2016), pp. 1002-1011. 
[4] O. Ciftci, M. Mehrtash, and A. Kargarian. "Data-driven nonparametric chance-constrained optimization for microgrid energy management". IEEE Transactions on Industrial Informatics, vol. 16, no. 4 (2020), pp. 2447-2457.

[5] H. Kanchev, D. Lu, F. Colas, V. Lazarov, and B. Francois. "Energy management and operational planning of a microgrid with a PV-based active generator for smart grid applications". IEEE Transactions on Industrial Electronics, vol. 58, no. 10 (2011), pp. 4583-4592.

[6] J. Lagorse, D. Paire, and A. Miraoui. "A multi-agent system for energy management of distributed power sources". Renewable Energy, vol. 35, no. 1 (2010), pp. 174-182.

[7] B. Papari, C. S. Edrington, I. Bhattacharya, and G. Radman. "Effective energy management of hybrid AC-DC microgrids with storage devices". IEEE Transactions on Smart Grid, vol. 10, no. 1 (2019), pp. 193-203.

[8] B. Papari, C. S. Edrington, and D. Gonsoulin. "Optimal energy-emission management in hybrid AC-DC microgrids with vehicle-2-grid technology". Journal of Renewable and Sustainable Energy, vol. 11, no. 1 (2019).

[9] B. Papari, C. S. Edrington, T. V. Vu, and F. Diaz-Franco. "A heuristic method for optimal energy management of DC microgrid". 2017 IEEE Second International Conference on DC Microgrids (ICDCM), pp. 337-343.

[10] T. Mühlpfordt, X. Dai, A. Engelmann, and V. Hagenmeyer. "Distributed power flow and distributed optimization-Formulation, solution, and open source implementation". Sustainable Energy, Grids and Networks, vol. 26 (2021).

[11] S. Boyd, N. Parikh, and E. Chu. "Distributed optimization and statistical learning via the alternating direction method of multipliers". Now Publishers Inc (2011).

[12] T. Erseghe. "Distributed optimal power flow using ADMM". IEEE Transactions on Power Systems, vol. 29, no. 5 (2014), pp. 2370-2380.

[13] S. Mhanna, G. Verbič, and A. C. Chapman. "Adaptive ADMM for distributed AC optimal power flow". IEEE Transactions on Power Systems, vol. 34, no. 3 (2019), pp. 2025-2035.

[14] Y. Zhang, M. Hong, E. Dall'Anese, S. V. Dhople, and Z. $\mathrm{Xu}$. "Distributed controllers seeking AC optimal power flow solutions using ADMM". IEEE Transactions on Smart Grid, vol. 9, no. 5 (2018), pp. 4525-4537.

[15] A. Engelmann, Y. Jiang, T. Mühlpfordt, B. Houska, and T. Faulwasser. "Toward distributed OPF using ALADIN". IEEE Transactions on Power Systems, vol. 34, no. 1 (2019), pp. 584594.

[16] S. Xia, S. Bu, C. Wan, X. Lu, K. W. Chan, and B. Zhou. "A fully distributed hierarchical control framework for coordinated operation of DERs in active distribution power networks". IEEE Transactions on Power Systems, vol. 34, no. 6 (2019), pp. 5184-5197.

[17] A. Supponen, A. Rautainen, J. Markkula, A. Mäkinen, P. Järventausta, and S. Repo. "Power quality in distribution networks with electric vehicle charging - a research methodology based on field tests and real data". 2016 Eleventh International Conference on Ecological Vehicles and Renewable Energies (EVER). 2016, pp. 1-11.

[18] R. Li, Q. Wu, and S. S. Oren. "Distribution locational marginal pricing for optimal electric vehicle charging management". IEEE Transactions on Power Systems, vol. 29, no. 1 (2014), pp. 203-211.

[19] J. Quiros-Tortos, L. F. Ochoa, and T. Butler. "How electric vehicles and the grid work together: Lessons learned from one of the largest electric vehicle trials in the world". IEEE Power and Energy Magazine, vol. 16, no. 6 (2018), pp. 64-76.

[20] Electric Vehicle Database. All electric vehicles. URL: https: //ev-database.org/. (accessed: 22.08.2020).

[21] Argonne National Laboratory Energy Systems Division. Light duty electric drive vehicles monthly sales updates. URL: https: //www.anl.gov/es/light-duty-electric-drive-vehicles-monthlysales-updates. (accessed: 22.08.2020).

[22] M. G. Flammini, G. Prettico, A. Julea, G. Fulli, A. Mazza, and G. Chicco. "Statistical characterisation of the real transaction data gathered from electric vehicle charging stations". Electric Power Systems Research, vol. 166 (2019), pp. 136-150.

[23] F. Pallonetto, M. Galvani, A. Torti, and S. Vantini. "A framework for analysis and expansion of public charging infrastructure under fast penetration of electric vehicles". World Electric Vehicle Journal, vol. 11, no. 1 (2020). ISSN: 2032-6653. URL: https://www.mdpi.com/2032-6653/11/1/18.

[24] P. Weldon, P. Morrissey, J. Brady, and M. O'Mahony. "An investigation into usage patterns of electric vehicles in Ireland". Transportation Research Part D: Transport and Environment, vol. 43 (2016), pp. 207-225.

[25] J. Lavaei, S. H. Low. "Zero duality gap in optimal power flow problem". IEEE Transactions on Power Systems, vol. 27, no. 1 (2012), pp. 92-107.

[26] S. H. Low. "Convex relaxation of optimal power flow-part I: formulations and equivalence". IEEE Transactions on Control of Network Systems, vol. 1, no. 1 (2014), pp. 15-27.

[27] P. H. Hoang, C. S. Edrington, B. Papari, G. Ozkan, and H. Ahn. "Distributed constrained optimization over networked systems via a singular perturbation method and application to economic dispatch". 2020 Clemson University Power Systems Conference (PSC), pp. 1-6.

[28] P. H. Hoang, G. Ozkan, P. R. Badr, B. Papari, and C. S. Edrington. "Distributed constrained optimization by singular perturbation method and its application to energy networks by real-time implementation". Sustainable Energy, Grids and Networks, vol. 27 (2021).

[29] M. E. Baran, F. F. Wu. "Network reconfiguration in distribution systems for loss reduction and load balancing". IEEE Transactions on Power Delivery, vol. 4, no. 2 (1989), pp. 1401-1407.

[30] W. I. Rowen. "Simplified mathematical representations of heavy-duty gas turbines". Journal of Engineering for Power, vol. 105 , no. 4 (1983), pp. 865-869.

[31] S. Grillo, S. Massucco, A. Morini, A. Pitto, and F. Silvestro. "Microturbine control modeling to investigate the effects of distributed generation in electric energy networks". IEEE Systems Journal, vol. 4, no. 3 (2010), pp. 303-312.

[32] A. D. Hansen, I. D. Margaris. "Type IV wind turbine model". DTU Wind Energy (2014).

[33] N. Femia, G. Petrone, G. Spagnuolo, and M. Vitelli. "A technique for improving P\&O MPPT performances of doublestage grid-connected photovoltaic systems". IEEE Transactions on Industrial Electronics, vol. 56, no. 11 (2009), pp. $4473-4482$.

[34] I. Richardson, M. Thomson, D. Infield, and C. Clifford. "Domestic electricity use: A high-resolution energy demand model". Energy and Buildings, vol. 42, no. 10 (2010), pp. $1878-1887$.

[35] J. Ponoćko, J. V. Milanović. "Forecasting demand flexibility of aggregated residential load using smart meter data". IEEE Transactions on Power Systems, vol. 33, no. 5 (2018), pp. 5446-5455.

[36] B. Johnson. "Power factor correction design for on-board chargers in electric vehicles". Texas Instruments' Application Report (2018).

[37] J. Wang, G. R. Bharati, S. Paudyal, O. Ceylan, B. P. Bhattarai, and K. S. Myers. "Coordinated electric vehicle charging with reactive power support to distribution grids". IEEE Transactions on Industrial Informatics, vol. 15, no. 1 (2019), pp. 5463.

[38] Global petrol prices. United Kingdom gasoline price. URL: https : / / www . globalpetrolprices . com / United - Kingdom / gasoline_prices/. (accessed: 24.08.2020). 


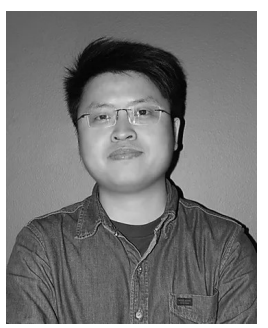

Phuong H. Hoang received his BS degree in Control and Automation Engineering in 2013 at Hanoi University of Science and Technology, Vietnam. He then obtained an MS degree in Mechanical Engineering at Gwangju Institute of Science and Technology in 2018. He researched distributed control and optimization applied to energy networks during his master's studies. After earning the MS degree, he was accepted to the Ph.D. program in Electrical Engineering Fall 2018 at Florida State University where he worked as a Graduate Research Assistant in Center for Advanced Power Systems under the supervision of Dr. Edrington. Currently, he is with Clemson University and also under the supervision of Dr. Edrington in the Department of Electrical and Computer Engineering. His current research interests include real-time CHIL simulations, energy management, and degradation forecasting for power systems.

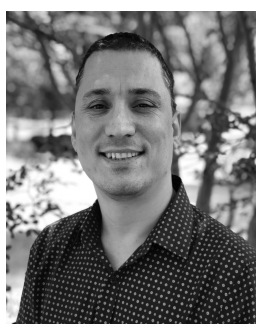

Gokhan Ozkan received his BS degrees in Teacher Training in Electrical Field and Energy System Engineering from Marmara University and Erciyes University, Turkey in 2006 and 2014, and his MS in Energy System Engineering from Erciyes University, Turkey in 2016. He was a lecturer at Bozok University, Turkey. He completed his $\mathrm{PhD}$ in Electrical and Computer Engineering at FAMU-FSU College of Engineering, and worked as a graduate research assistant at the Center for Advanced Power Systems, Florida State University. He is now working as a Post-doctoral Research Associate for RT-COOL (Real-Time COntrol and Optimization Laboratory) at Clemson University. His research interests include renewable energy real-time modelling, simulation, control design, and electrothermal management for power converters.

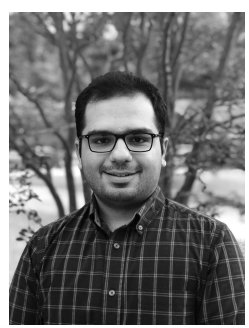

Payam Ramezani Badr received his B.S. degree in electrical engineering from the University of Lorestan, Iran, in 2019 and is currently pursuing his $\mathrm{PhD}$ degree as a direct-entry candidate in electrical engineering with a focus on adaptive control systems at Clemson University. He works as a research assistant for RT-COOL (Real-Time COntrol and Optimization Laboratory) in Holcombe Department of Electrical and Computer Engineering (ECE), Clemson University under the supervision of Dr. Chris S. Edrington. His research interests include microgrids, control, performance optimization, electro-thermal modelling of machines, and real-time experimentation. Payam Badr is a committee member for IEEE Student Branch Clemson University. He also currently serves as a senator in the Graduate Student Government at Clemson University representing the ECE department.

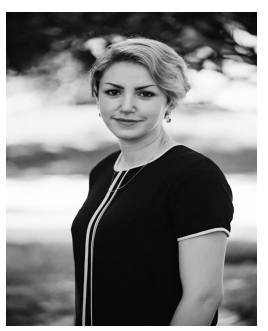

Behnaz Papari received her Ph.D. degree in electrical and electronics engineering from Florida State University, USA in 2018. She is currently an Assistant Professor in Power Electronics Engineering, and manager of the Duke Energy Smart Grid Lab. She is associated with the Energy Production and Infrastructure Center. She has expertise in power systems with an emphasis on modeling, analysis, control, planning, and optimization. Her specialties are distributed controls and decision analysis under uncertainty, applications on energy system modeling, and Stochastic optimization. Her research interests include renewable energy sources, power and energy management, control of stand-alone and utilityinteractive energy systems, distributed control of smart grids, stochastic analysis, and real-time power distribution system simulation and hardwarein-the-loop instantiation.

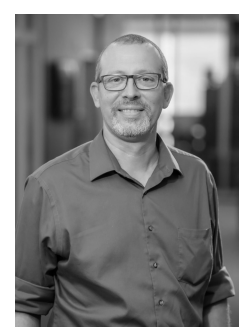

Christopher Shannon Edrington received his BS in Engineering from Arkansas State University in 1999 and his MS and PhD in Electrical Engineering from the Missouri University of Science and Technology in 2001 and 2004, respectively; where he was both a DoE GAANN Fellow, NSF IGERT Fellow and Grainger Foundation Fellow. He currently is the Warren H. Owen Distinguished Professor of Electrical and Computer Engineering at Clemson University and is the lead for RT-COOL (Real-Time COntrol and Optimization Laboratory) as well as the co-director of the Smart Energy and Propulsion Focus Area in the new VIPRGS (VIrtual PRototyping for Ground vehicle Systems) Center. His research interests include modeling, simulation, and control of electromechanical drive systems; applied power electronics; distributed control; integration of renewable energy, storage, and pulse power loads. Dr. Edrington has published over 170 papers (including 2 IEEE Prize Awards and multiple conference paper awards), has graduated $23 \mathrm{MS}$ students and $16 \mathrm{PhD}$ students (with 3 in process) and has 6 patents (in real-time stability and complexity metrics).

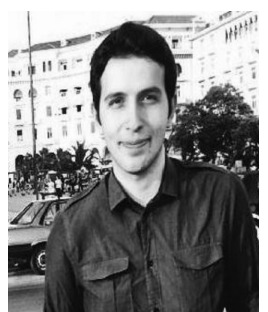

Mustafa Alparslan Zehir received his BS in 2010, MS in 2013 and $\mathrm{PhD}$ in 2019 in Electrical Engineering at Istanbul Technical University (ITU). From 2012 to 2019 , he worked as a research and teaching assistant at ITU, while also taking part in a number of European projects. He is currently a postdoctoral researcher at MaREI/Environmental Research Institute, University College Cork and he is in the team of CREDENCE international project. His research fields are electric power distribution, distributed energy resources, demand response, electricity markets, prosumers and microgrids.

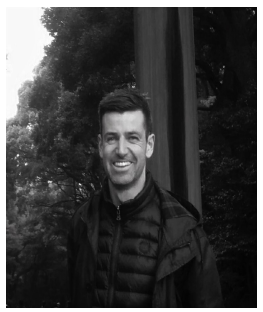

Barry Hayes is a researcher and lecturer in Electrical and Electronic Engineering at UCC. His research interests include the grid integration of sustainable energy technologies, and the operation and planning of future power systems.

Before joining UCC in July 2018, Barry was a lecturer in Electrical and Electronic Engineering at NUI Galway. From 2013 to 2016, Barry was a Marie Sklodowska-Curie research fellow at IMDEA Energy in Madrid, where he worked on European and national research projects related to power systems and smart grids. During his time in Madrid, he was awarded the Jose Castillejo and Juan de la Cierva grants from the Spanish national research councils.

$\mathrm{He}$ holds a $\mathrm{PhD}$ in Electrical Power Systems Engineering from the University of Edinburgh (2013), and has held visiting researcher positions at National Grid UK (2011) and at the University of Tennessee (2016). Barry has more than 30 peer-reviewed international publications in book chapters, conferences, and journals including IEEE Transactions on Power Systems and IEEE Transactions on Smart Grid. He is a Senior Member of the IEEE.

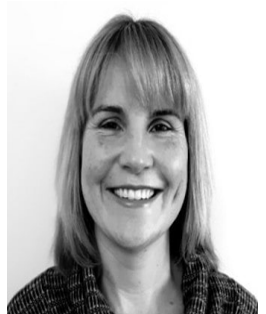

Laura Mehigan is a final year $\mathrm{PhD}$ Researcher in the Energy Policy and Modelling team in MAREI. Her research focuses on understanding the future shape of electricity systems and more specifically the interplay between decentralization and interconnection as part of the CREDENCE project. She joined in 2017 having spent the previous 12+ years working in a variety of senior engineering roles with EirGrid, the Irish Transmission System Operator. She holds a postgraduate diploma in Project Management from Trinity College Dublin and a Degree in Electrical and Electronic Engineering from University College Cork. 
1

2

3

4

5

6

7

8

9

10

11

12

13

14

15

16

17

18

19

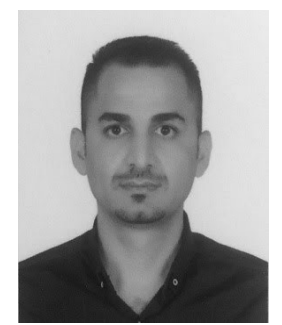

Dlzar Al Kez is currently a PhD student at Queen's University Belfast. Before joining Queen's University Belfast, he obtained an MS in Energy and Sustainability with Electrical Power Engineering at University of Southampton and a BS in Electrical Engineering at University of Sulaimani. His research interests include impact of distributed generation on the power system dynamics, impact of renewable energy on the power system dynamics, and Application of energy storage, smart appliances, and FACTS devices in power system stability.

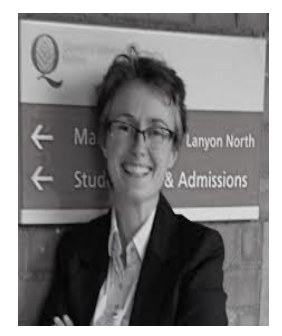

Aoife M. Foley (M'06) received the B.Eng. (Hons.) degree in civil engineering from the University College Cork, Cork, Ireland, in 1996, the M.Sc. degree in environmental \& transportation engineering from Trinity College, Dublin, Ireland, in 1999, and the $\mathrm{Ph} . \mathrm{D}$. degree in energy engineering from the University College Cork in 2011. She worked in industry until 2008. She is currently a Reader with the School of Mechanical and Aerospace Engineering, Queen's University Belfast, Belfast, U.K. Her research interests include wind power, energy markets, energy storage, and electric vehicles. Dr. Foley has been a Chartered Engineer since 2001 and a Fellow of Engineers Ireland since 2012. She is the Editor-in-Chief of Renewable and Sustainable Energy Reviews. 